\title{
Conversion chemotherapy followed by hepatic resection in colorectal cancer with initially unresectable liver-limited metastases
}

\author{
GENNARO GALIZIA $^{1 *}$, FERDINANDO DE VITA ${ }^{2 *}$, EVA LIETO $^{1}$, ANNA ZAMBOLI ${ }^{1}$, FLORIANA MORGILLO ${ }^{2}$, \\ PAOLO CASTELLANO $^{1}$, ANDREA MABILIA ${ }^{1}$, ANNAMARIA AURICCHIO ${ }^{1}$, ANDREA RENDA $^{3}$, \\ FORTUNATO CIARDIELLO ${ }^{2}$ and MICHELE ORDITURA ${ }^{2}$
}

\author{
Divisions of ${ }^{1}$ Surgical Oncology and ${ }^{2}$ Medical Oncology, 'F. Magrassi - A. Lanzara' Department of Clinical \\ and Experimental Medicine and Surgery, Second University of Naples School of Medicine, Naples; \\ ${ }^{3}$ Division of General Surgery, Federico II University of Naples School of Medicine, Naples, Italy
}

Received June 18,2013; Accepted August 26, 2013

DOI: $10.3892 / o r .2013 .2795$

\begin{abstract}
The best management choice in colorectal cancer patients with unresectable liver-only metastases should be represented by conversion chemotherapy aiming to reduce liver cancer deposits, thereby permitting curative surgery. Forty-eight consecutive stage IV colorectal cancer patients were treated with different chemotherapeutic regimens including biological drugs. Objective responses to chemotherapy were seen in 27 patients (56.2\%; 95\% CI 42.1-70.2\%). Four patients $(8.3 \%)$ showed complete response, 23 patients (47.9\%) partial and 13 patients (27.1\%) stable response. Eight patients (16.7\%) progressed. The conversion rate was $35.4 \%$ (95\% CI 21.8-48.9\%) with 17 patients suitable for liver resection. Four complete responder patients refused surgery. The remaining 13 patients underwent curative hepatic resection (resection rate $27.1 \%$; 95\% CI 14.5-39.6\%). The likelihood of a successful conversion chemotherapy appeared significantly related to the best response and to the K-Ras status. Wild-type K-Ras patients undergoing cetuximab therapy showed the best conversion rate. The four-year survival rate was significantly enhanced in converted compared to non-converted patients (57.1 and 0\%, respectively), and in resected compared to non-resected patients (53.3 and $10.1 \%$, respectively). Synchronous metastases and no conversion were shown to be the only covariates independently associated with a poorer
\end{abstract}

Correspondence to: Dr Michele Orditura, Division of Medical Oncology, 'F. Magrassi - A. Lanzara' Department of Clinical and Experimental Medicine and Surgery, Second University of Naples School of Medicine, c/o II Policlinico, Edificio 3, Via Pansini 5, I-80131 Naples, Italy

E-mail: michele.orditura@unina2.it

*Contributed equally

Key words: colorectal cancer, liver metastasis, conversion chemotherapy long-term outcome. The possibility of curative liver surgery significantly prolongs outcome for colorectal cancer patients with unresectable liver-limited metastases. Prospective randomized trials are required to define the conversion rates with biological drugs.

\section{Introduction}

Colorectal cancer (CRC) is the third most prevalent cancer in the world with more than 940,000 cases and nearly 500,000 deaths occurring annually worldwide (1). Almost $20-25 \%$ of colorectal cancer patients present with distant metastases at the time of diagnosis and the median overall survival (OS) does not exceed 2 years (2). The liver is involved in $80-90 \%$ of the cases and, in almost $50 \%$, it is the only site of metastasis. Integration of chemotherapy and surgery in the treatment of hepatic metastases represents the best choice for improving survival in this subset of patients (3). Notably, 10-30\% of newly diagnosed colorectal cancer patients with liver-only deposits have their metastases resectable at presentation. In such instances, the European Colorectal Metastases Treatment Group strongly recommends pre-operative chemotherapy and liver resection (4). However, an estimated $70-90 \%$ of these patients have unresectable hepatic metastases at presentation (5). Until a few years ago, the limited clinical efficacy of available anticancer drugs coupled with no substantial improvements in liver surgery, which constituted a critical procedure with high postoperative mortality and morbidity rates, did not allow for a consistent chance of cure to these patients. In the last decade, there has been a shift in this paradigm mainly due to a more anatomic and surgical approach since the successful introduction of liver resection, as well as the clinical development of more effective drugs, such as oxaliplatin and irinotecan, and more recently, of targeted monoclonal antibodies. There is increasing evidence that the best management choice in colorectal patients with unresectable liver-only metastases should be represented by integrated treatments in which the critical aim of chemotherapy is to reduce hepatic cancer deposits, thus permitting potentially 
curative liver surgery (known as conversion chemotherapy), rather than to represent a palliative support program (6). In particular, standard 5-fluorouracil plus irinotecan or oxaliplatin-based chemotherapy offers conversion rates ranging from 9 to $22 \%$ according to the GERCOR trial with a potentially curative liver resection (known as $\mathrm{R} 0$ resection) rate ranging from 7 to $13 \%$ (7). However, although there is a strong correlation between response rate and resection rate both in patients selected for liver metastases only and in non-selected patients, data on conversion rates with biological regimens are scarce and conflicting, and no randomized trials focusing on a liver-limited disease population are available (8). In addition, duration of treatment, drug toxicity, particularly related to liver functions, and optimal timing for liver surgery remain unclear (6).

In the present study, we report on treatment of 48 colorectal cancer patients with unresectable metastases confined to the liver, with conversion chemotherapy with or without anti-epidermal growth factor receptor (EGFR-cetuximab) or anti-vascular endothelial growth factor (VEGF-bevacizumab) monoclonal antibody. The study end-points included assessment of: (i) conversion and liver R0 resection rates; (ii) relationship between conversion rate and other prognostic factors including different chemotherapeutic regimens and (iii) OS rates in converted and non-converted patients.

\section{Patients and methods}

Clinical records from 48 consecutive patients with stage IV colorectal cancer and unresectable liver-only metastases were retrospectively selected from the database of our Department in the period from December, 2006 to February, 2011. All tumors were staged upon evaluation of findings of physical examination, routine laboratory tests and diagnostic imaging [complete colonoscopy with biopsy, abdominal ultrasound, chest and abdomen CT-scan, scintigraphic bone scan, whole body magnetic resonance imaging and F-18 fluorodeoxyglucose positron emission tomography (FDG-PET) scan, and contrast enhanced ultrasonography of the liver when appropriate].

All patients had hepatic metastases judged unresectable due to invasion of major liver pedicles (metastatic disease adjacent or involving all 3 hepatic veins, and/or the portal vein bifurcation, and/or the retrohepatic vena cava, and/or the vascular structures of the opposite lobe) or intrahepatic dissemination (bilobar disease) requiring a liver resection potentially capable of jeopardizing postoperative liver function. The inoperability was defined by a multidisciplinary team including two independent skillful liver surgeons blinded each other. All cases were independently reviewed by each surgeon; discrepancies between investigators ( $<10 \%$ of the cases) required a third joint observation with conclusive agreement.

Thirteen patients $(27.1 \%)$ showed metachronous liverlimited metastases following a potentially curative colorectal cancer resection (means 20 \pm 8 , range 13-42, median 17 months from colorectal surgery). The remaining 35 patients presented synchronous liver metastases; all but 2 underwent immediate surgery of the primary colorectal cancer due to significant tumor symptoms (20 patients), or according to our own policy in asymptomatic patients (9). Overall, $46 / 48$ patients $(96 \%)$ underwent resection of the primary colorectal cancer.
Before the start of conversion chemotherapy, patients were screened for K-Ras status; 24 (50\%) harbored a wild-type and $21(43.7 \%)$ a mutated protein. In 3 cases $(6.3 \%)$, K-Ras status was not evaluated. All 24 wild-type K-Ras cancer patients were treated with irinotecan or oxaliplatin-based chemotherapy and anti-EGFR antibody cetuximab. Sixteen patients received as first-line treatment irinotecan or oxaliplatin and 5-fluorouracil or capecitabine-based chemotherapy and anti-VEGF antibody bevacizumab. Finally, 8 patients received chemotherapy without biological drugs. Response to systemic therapy was evaluated every two months according to RECIST guidelines; metastases were restaged with regard to their resectability and, when appropriate, patients were referred to liver surgery as soon as possible, albeit not sooner than 4 weeks after the last cycle of chemotherapy. A switch to second-line chemotherapy was decided in cases of disease progression (PD). No patient was lost to follow-up and it was completed by May 31, 2011. All patients gave their informed consent and the study was approved by the Department of Clinical and Experimental Medicine and Surgery of the Second University of Naples.

Statistical analysis. Statistical analysis was carried out using the SPSS statistical package (SPSS Inc., Chicago, IL, USA) integrated by the MedCalc ${ }^{\circledR}$ software version 9.4.2.0 (Mariakerke, Belgium). In all analyses, the significance level was specified as $p<0.05$. Logistic regression analysis was performed to determine the relationship between conversion rate and other potentially influential covariables. The following prognostic factors were considered: age, gender, site and tumor (T) and nodes $(\mathrm{N})$ of the colorectal tumor, time of liver metastasis appearance (synchronous or metachronous), K-Ras status, type of conversion chemotherapy and best response. The OS was calculated from the date of initiation of the conversion chemotherapy to the date of mortality or the end of followup, marking alive patients as censored. Univariate statistical analysis was determined by long-rank test (Mantel-Cox). Curves were plotted using the product-limit method (KaplanMeier); p-values and hazard ratios (HR) with $95 \%$ confidence interval (CI) were provided. The independent significance of prognostic variables, showing a p-value $<0.1$ on univariate analysis, was determined by multivariate analysis using the Cox's proportional hazards model. The model was also tested for possible interaction effects between covariates correlated with each other, to determine if these variables could independently influence survival. Finally, a stepwise multivariate analysis was performed in order to generate a model of the best linear combination of variables able to predict OS rate.

\section{Results}

Objective responses to conversion chemotherapy were seen in 27 patients $(56.2 \%$; 95\% CI 42.1-70.2\%). Four patients $(8.3 \%)$ achieved radiological complete response (CR) of liver metastases. Twenty-three patients $(47.9 \%)$ showed a radiological partial response (PR). On the contrary, 13/48 patients (27.1\%) were considered stable (SD) and continued chemotherapy until progression or unacceptable toxicity, and 8 patients $(16.7 \%)$ progressed during first-line treatment. Fig. 1 shows the flow-chart of all eligible patients. Overall, 17 patients showed a significant reduction in liver metastases so as to 


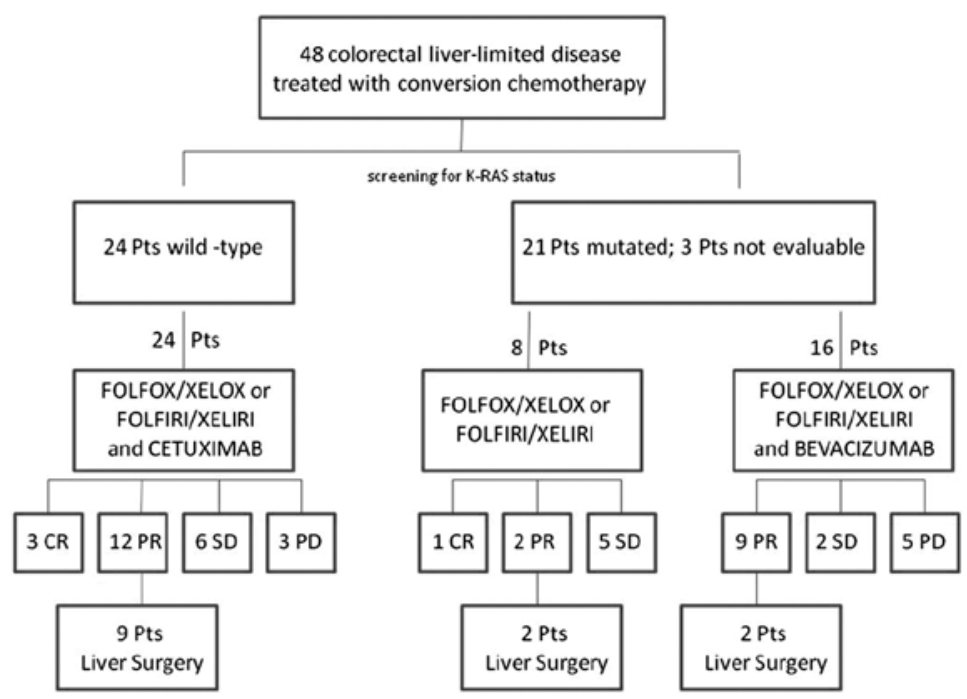

Figure 1. Flow-chart of 48 eligible patients. CR, complete response; PR, partial response; SD, stable disease; PD, progressive disease FOLFIRI, (day 1, irinotecan $180 \mathrm{mg} / \mathrm{m}^{2}$, folinic acid $400 \mathrm{mg} / \mathrm{m}^{2}$, fluorouracil $400 \mathrm{mg} / \mathrm{m}^{2}$ i.v. bolus, then $2,400 \mathrm{mg} / \mathrm{m}^{2}$ over $46 \mathrm{~h}$ continuous infusion, every 2 weeks); XELIRI, (day 1 , irinotecan $250 \mathrm{mg} / \mathrm{m}^{2}$, capecitabine $1,700 \mathrm{mg} / \mathrm{m}^{2} /$ day bid os for 14 days followed by 7 days rest, every 3 weeks); FOLFOX, day 1, oxaliplatin $85 \mathrm{mg} / \mathrm{m}^{2}$, folinic acid $400 \mathrm{mg} / \mathrm{m}^{2}$, fluorouracil $400 \mathrm{mg} / \mathrm{m}^{2}$ i.v. bolus, then $2,400 \mathrm{mg} / \mathrm{m}^{2}$ over $46 \mathrm{~h}$ continuous infusion, every 2 weeks); XELOX, (day 1, oxaliplatin $130 \mathrm{mg} / \mathrm{m}^{2}$, capecitabine $1,700 \mathrm{mg} / \mathrm{m}^{2} / \mathrm{day}$ bid os for 14 days followed by 7 days rest, every 3 weeks); CETUXIMAB, initial dose of $400 \mathrm{mg} / \mathrm{m}^{2}$ in $2 \mathrm{~h}$ i.v. infusion, and then $250 \mathrm{mg} / \mathrm{m}^{2}$ in $1 \mathrm{~h}$ i.v. infusion weekly; BEVACIZUMAB, $5 \mathrm{mg} / \mathrm{kg}$ i.v. infusion on day 1 every 2 weeks and stopped 4 weeks before surgery.

become amenable to resection (conversion rate $35.4 \%$; $95 \% \mathrm{CI}$ 21.8-48.9\%). However, the 4 complete response patients refused liver surgery $(2$ are alive without cancer at 8 and 21 months from the end of chemotherapy, and the remaining 2 patients relapsed in the liver at 12 and 13 months after chemotherapy, and are currently awaiting liver resection). The remaining 13 patients (all partial response patients) underwent hepatic resection after shrinkage of initially unresectable liver metastases (liver resection rate 27.1\%; 95\% CI 14.5-39.6\%). Liver operations, including 9 major hepatectomies ( $\geq 3$ liver segments) and 4 bilateral and multiple metastasectomies, were performed through an exclusive abdominal incision, with lymphadenectomy of the hepatic pedicle followed by liver transection performed by Kelly clamp crushing method or, more recently, using a new fully automated radiofrequency generator supplying a comb-shaped bipolar multi-electrode device [SURTRON ${ }^{\circledR}$ SB, LED SpA, Aprilia (LT, Italy)] (10). Inflow vascular occlusion was never necessary. All procedures were judged potentially curative ( $\mathrm{R} 0$ resection), with removal of all macroscopic tumoral mass(es), absence of microscopic residual tumor, and histology-negative resection margins. No postoperative hemorrhage, biliary leakage or abscess formation were observed. One patient suffered from slight liver impairment function successfully treated with medical therapy. All resected patients underwent postoperative chemotherapy according to the previous regimen permitting liver surgery.

The median number of chemotherapy cycles was 8 (means $8.7 \pm 4.1$; range $3-23$ ). In converted patients, the number of cycles of chemotherapy (means 7.0 3.2 ; range $3-13$, median 6) was lower than in non-converted patients (means 9.6 \pm 4.2 ; range 4-23, median 8). The difference was statistically significant in logistic regression analysis, as shown in Fig. 2 ( $\mathrm{r}=0.3022$, slope $=-2.5541, \mathrm{p}=0.0370)$.

The likelihood of a successful conversion chemotherapy was not related to age, gender, site of the primary colorectal

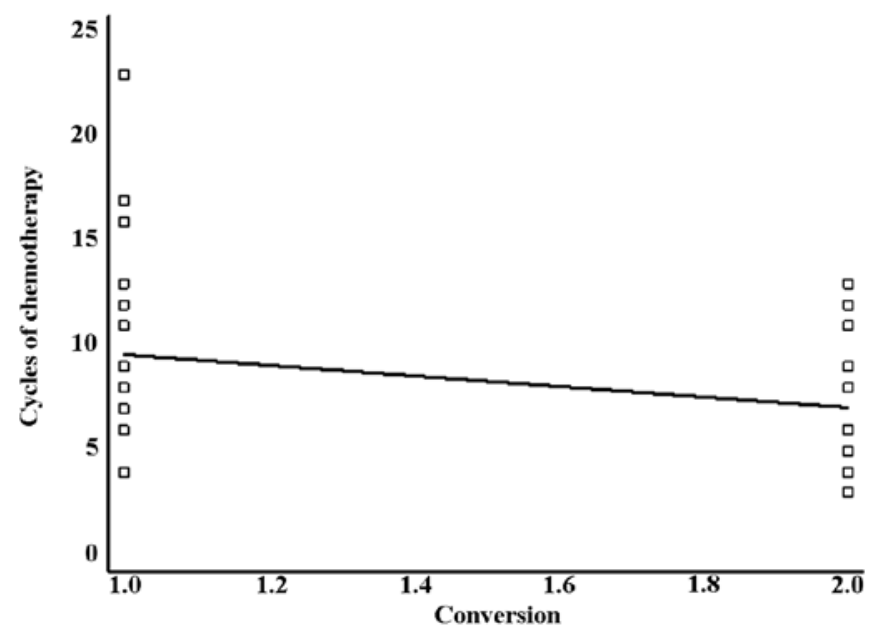

Figure 2. Linear correlation between number of cycles of chemotherapy and conversion rate. Converted patients required a significantly lower number of cycles of chemotherapy than non-converted patients.

tumor, $\mathrm{T}$ and $\mathrm{N}$ parameters, time of liver metastasis appearance and type of chemotherapy. On the contrary, it appeared significantly related to the best response [all complete and $13 / 23$ partial response patients showed conversion $(r=0.6467$, slope $=-1.1708, \mathrm{p}<0.0001)]$, and to the K-Ras status. Indeed, $12 / 24$ wild-type K-Ras patients $(50 \%)$ were suitable for liver resection at the end of conversion chemotherapy, while only $5(20.8 \%)$ of the remaining 24 patients (21 mutated and 3 not evaluable K-Ras patients) had a successful response $(r=0.3047$, slope $=-0.3188, \mathrm{p}=0.0350$ ).

Analysis related to overall survival. The mean follow-up time was $17 \pm 10$ months (range $3-54$, median 13 months). During this time period, 21 patients $(43.7 \%)$ succumbed to the disease. One to four-year OS rates were 78.8, 50.1, 29.2 and $19.5 \%$, 
Table I. Characteristics of the series and univariate analysis related to survival rate.

\begin{tabular}{|c|c|c|c|c|c|c|}
\hline Characteristics & No. of pts & Deceased & $\begin{array}{l}\text { 48-month } \\
\text { survival (\%) }\end{array}$ & Hazard ratio & $\begin{array}{l}95 \% \mathrm{CI}^{\mathrm{a}} \\
\text { Hazard ratio }\end{array}$ & P-value \\
\hline 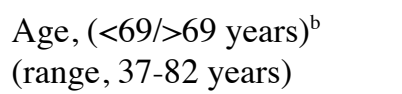 & $24 / 24$ & $12 / 9$ & $19 / 0$ & 1.40 & $0.59-3.33$ & 0.4346 \\
\hline Gender (male/female) & $27 / 21$ & $14 / 7$ & $13 / 34$ & 0.85 & $0.32-2.19$ & 0.7342 \\
\hline $\begin{array}{l}\text { Site }(\text { right/left/rectum) } \\
\text { Right vs. no right }\end{array}$ & $\begin{array}{c}18 / 23 / 7 \\
18 / 30\end{array}$ & $\begin{array}{c}3 / 14 / 4 \\
3 / 18\end{array}$ & $\begin{array}{c}63 / 12 / 0 \\
63 / 8\end{array}$ & $\begin{array}{c}- \\
0.32\end{array}$ & $\begin{array}{c}- \\
0.16-1.02\end{array}$ & $\begin{array}{l}0.1667 \\
\mathbf{0 . 0 5 6 7}\end{array}$ \\
\hline Synchronous/metachronous & $35 / 13$ & $16 / 5$ & $19 / 51$ & 0.43 & $0.08-1.20$ & 0.0917 \\
\hline \multicolumn{7}{|l|}{ Tumor $^{\mathrm{d}}$} \\
\hline 2 & 4 & 0 & 100 & - & - & 0.2856 \\
\hline 3 & 28 & 12 & 0 & & & \\
\hline 4 & 14 & 8 & 19 & & & \\
\hline \multicolumn{7}{|l|}{ Nodes $^{\mathrm{d}}$} \\
\hline 0 & 14 & 5 & 0 & - & - & 0.1021 \\
\hline 1 & 19 & 7 & 23 & & & \\
\hline 2 & 13 & 8 & 19 & & & \\
\hline \multicolumn{7}{|l|}{ K-Ras status } \\
\hline Wild-type & 24 & 6 & 48 & - & - & $<0.0001$ \\
\hline Mutated & 21 & 13 & 9 & & & \\
\hline Not determined & 3 & 2 & 0 & & & \\
\hline WT vs. no WT & $24 / 24$ & $6 / 15$ & $48 / 8$ & 0.41 & $0.18-1.01$ & 0.0554 \\
\hline Chemotherapy & 8 & 5 & 0 & - & - & 0.1388 \\
\hline Chemo & & & & & & \\
\hline Chemo + cetuximab & 24 & 6 & 48 & & & \\
\hline Chemo + bevacizumab & 16 & 10 & 12 & & & \\
\hline \multicolumn{7}{|l|}{ Best response to chemo } \\
\hline Complete & 4 & 0 & 100 & - & - & 0.0033 \\
\hline Partial & 23 & 7 & 35 & & & \\
\hline Stable disease & 13 & 8 & 0 & & & \\
\hline Progression disease & 8 & 6 & 0 & & & \\
\hline \multicolumn{7}{|l|}{ Conversion } \\
\hline Yes & 17 & 2 & 57 & 0.09 & $0.06-0.37$ & $<0.0001$ \\
\hline No & 31 & 19 & 0 & & & \\
\hline \multicolumn{7}{|l|}{ Liver resection } \\
\hline Yes & 13 & 2 & 53 & 0.16 & $0.09-0.63$ & 0.0037 \\
\hline No & 35 & 19 & 10 & & & \\
\hline
\end{tabular}

${ }^{\mathrm{a}} 95 \%$ confidence interval (CI) of hazard ratio of cancer-related mortality; ${ }^{\mathrm{b}}$ median value; ${ }^{\text {cright }}$ colon indicates cecum, ascending and proximal transverse colon; left colon indicates distal, transverse colon, descending and sigmoid colon until the sigma-rectum junction; ${ }^{\mathrm{d}} 46 / 48$ primary tumor resected patients; ${ }^{\mathrm{e}}$ chemo indicates irinotecan or oxaliplatin and 5-fluorouracil or capecitabine-based chemotherapy, without use of biologic drugs. Pts, patients.

respectively (means $27 \pm 3$, median 24 months). In univariate analysis, age, gender, stage of the primary colorctal cancer, and type of chemotherapy were not significantly associated with OS. On the contrary, the right site of the primary colorectal tumor, metachronous metastases, wild-type K-Ras, and objective response to chemotherapy were demonstrated to be significantly associated with an improved survival rate (Table I). Liver metastasis shrinkage and a further chance of potentially curative liver surgery as well as complete response were significantly correlated with better survival rates. One to four-year OS rates in 17 converted patients were $100,85.7,85.7$ and $57.1 \%$, which were significantly different from the rates recorded in non-converted patients $(67.4,32.4$ and $0 \%$ at 3 years, respectively). Although 4 converted patients did not undergo liver resection and are living, the curves of 13 resected patients plotted against non-resected patients still 
Table II. Multivariate analysis related to overall survival in 48 colon cancer patients treated with conversion chemotherapy for liver metastases.

\begin{tabular}{lccccr}
\hline Variables & Coefficient & Standard error & Hazard ratio & $\begin{array}{c}95 \% \mathrm{CI}^{\mathrm{a}} \\
\text { Hazard ratio }\end{array}$ & P-value \\
\hline Left colon and rectum & 0.9163 & 0.7514 & 2.49 & $0.57-10.82$ & 0.2227 \\
Synchronous metastases & 1.9543 & 0.7649 & 7.05 & $1.58-31.37$ & $\mathbf{0 . 0 1 0 6}$ \\
K-Ras mutated & 0.6741 & 0.4931 & 1.96 & $0.75-5.13$ & 0.1716 \\
No response to chemo & 0.0534 & 0.4102 & 1.05 & $0.47-2.34$ & 0.8963 \\
No conversion & 2.7426 & 1.2724 & 15.5 & $1.29-185.6$ & $\mathbf{0 . 0 3 1 1}$ \\
Conversion/K-Ras status $^{\mathrm{b}}$ & 0.4252 & 0.5088 & 2.31 & $0.54-9.95$ & 0.4034 \\
Conversion/response $^{\mathrm{b}}$ & 0.0106 & 0.4073 & 7.11 & $1.60-31.51$ & 0.9792 \\
\hline
\end{tabular}

Multivariate analysis was performed including variables with significant value on univariate analysis. ${ }^{\mathrm{a}} 95 \%$ confidence interval (CI) of hazard ratio of cancer-related mortality; banalysis with interaction. The interaction terms were not significant. This indicates that, regardless of K-Ras status and response to chemotherapy, conversion is an independent variable.

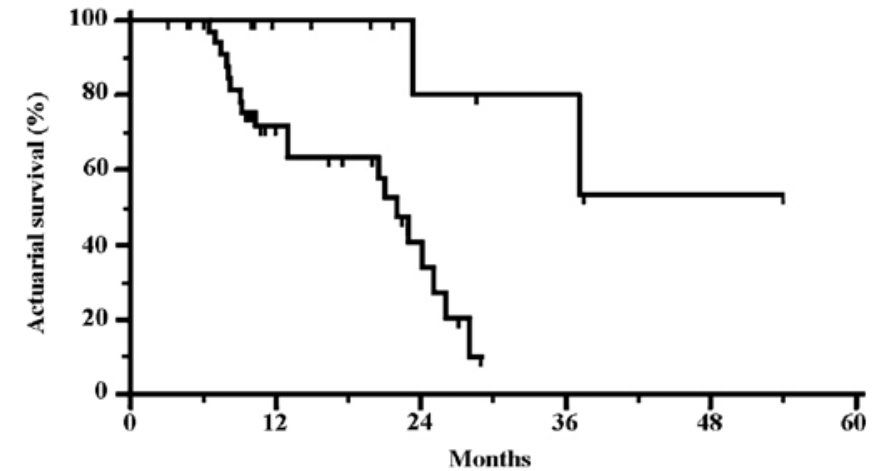

Number at risk

Group: 1

13

2

\section{8}

17

\begin{abstract}
4
\end{abstract}
6
1

0
1

0

Figure 3. Overall survival rates in 13 (group 1) colorectal cancer patients undergoing curative liver resection, and 35 (group 2) non-resected colorectal cancer patients showing unresectable liver metastases after the conversion chemotherapy (31 patients) or complete radiological response (4 patients refused surgery.

showed a significant difference. One to four-year OS rates were $71.6,40.6,10.1$ and $10.1 \%$ in non-resected and 100, 80, 80 and $53.3 \%$ in resected patients, respectively. The HR for OS was 0.16 (95\% CI $0.09-0.63 ; \mathrm{p}=0.0037)$, corresponding to a $70 \%$ increase in the rate of survival at 28 months from $10.1 \%$ (1.4-18.8\%) to $80 \%$ (62.1-97.9\%) with liver resection, and to an increase in the median survival from 22 to 54 months (Fig. 3). Of note, resected patients had an estimated $16 \%$ relative risk of cancer mortality compared to non-resected patients.

In multivariate analysis, metachronous metastases and conversion were shown to be the only covariates independently associated with a longer survival rate (Table II). Since conversion rate correlated with K-Ras status and response to chemotherapy (see above), thus questioning the true significance of this factor in statistical analyses, Cox's test was performed with activation of an interaction term. Independently of K-Ras status and response, possibility of conversion came out as an independent variable for long-term outcome. After backward

elimination, stepwise regression selected again synchronous metastases ( $\mathrm{HR}=5.67,95 \%$ CI 1.59-20.19, $\mathrm{p}=0.0077)$ and no conversion $(\mathrm{HR}=35.29,95 \% \mathrm{CI} 4.22-295.08, \mathrm{p}=0.0010)$ as the best combination of variables capable of predicting poor long-term OS.

\section{Discussion}

The standard of care for metastatic colorectal cancer consists of a combination of 5-fluorouracil, leucovorin and oxaliplatin or irinotecan-based chemotherapy; the different sequences of drugs were equivalent in terms of efficacy in a phase III randomized trial (7). Furthermore, exposure to all cytotoxic agents was shown to correlate with increase in survival (11). Recently, combination of conventional chemotherapy with agents targeting the vascular endothelial growth factor and epidermal growth factor receptor in the wild-type K-Ras population was also shown to add improvement in the chemotherapeutic efficacy (12-15). However, hepatic resection remains the only procedure to cure stage IV colorectal cancer patients with liverlimited metastases and, for immediately resectable disease, preoperative and perioperative chemotherapy has been shown to offer a longer progression-free and overall survival $(4,16,17)$.

Management of unresectable metastases of colorectal cancer is a significant challenge. Tumor response to chemotherapy has been clearly demonstrated to correlate with resectability rate. The efficacy of new regimens in this context may therefore be primarily evaluated with regard to their ability to induce tumor shrinkage, thus allowing surgery, rather than to longterm control of disease (18). Standard chemotherapy regimens offer conversion rates of between 9 and $22 \%$ with $\mathrm{R} 0$ resection rates of $7-13 \%$, and intensified chemotherapy with triplet combination, known as FOLFOXIRI, has been associated with overall response rates of $70 \%$ and $19 \%$ of R0 hepatic surgery in phase III randomized trials (19). Despite these relevant results, this regimen has not entered clinical practice due to substantial toxicity. Furthermore, several trials including an anti-EGFR antibody resulted in an increased response rate and a higher $\mathrm{R} 0$ resection rate. In particular, in the phase II randomized CELIM study, which combined cetuximab with FOLFIRI or 
FOLFOX, complete or partial responses in $62 \%$ of patients with a $34 \%$ of R0 resection were recorded (20). Furthermore, recently published phase II trials reported notable data on the ability of chemotherapy plus anti-VEGF antibody bevacizumab to shrink inoperable liver metastases (21-24).

In our series, we registered a rate of major responses equal to $56.2 \%$, which was not very distant from response rates from other trials in which chemotherapy and biologic drugs were delivered in selected populations of colorectal cancer with liver limited disease. However, best response was not an independent prognostic variable correlated with overall survival. This was not unexpected, since the main factor influencing long-term outcome was not related to tumor response, but to the likelihood to resect hepatic deposits. Among 27 responder patients, substantial shrinkage of liver metastases was obtained in 17 patients $(35.4 \%)$, and 13 of them (27.1\%) who underwent potentially curative surgery showed the best long-term outcome. Of note, 2 of 4 complete responder patients refusing surgery had liver recurrence, suggesting that complete radiological response was not able to predict complete tumoral necrosis, thus confirming the need for an aggressive surgical approach $(25,26)$.

As per the policy of our treating institution, all patients with unresectable metastases from colorectal cancer are treated with chemotherapy and anti-EGFR antibody if harboring wild-type K-Ras. Of note, although no different activity in terms of radiological response was seen between the addition of cetuximab (in wild-type K-Ras population) or bevacizumab (in mutated patients), $9(37.5 \%)$ of 24 patients treated with chemotherapy plus cetuximab could be subjected to resection, as opposed to only $12 \%$ in the chemotherapy plus bevacizumab population.

For patients with liver metastases that are resectable at presentation, perioperative chemotherapy has become the standard treatment in several institutions, with the recommendation that surgery be performed after a maximum of 6 cycles of systemic therapy (17). On the contrary, in the case of patients with initially unresectable liver metastases, there is no general agreement regarding the number of cycles of chemotherapy to be delivered. Therefore, it is recommended that patients be carefully monitored and surgery performed as soon as the metastases become resectable (18). However, excessive duration of chemotherapy aimed at shrinking hepatic deposits may produce substantial liver damage, as shown by several series reporting on associations between irinotecan and steatohepatitis and between oxaliplatin and sinusoidal dilatation (27), with significantly increased post-operative morbidity (28). However, it was recently shown that liver surgery can be safely performed in patients undergoing preoperative chemotherapy, and that addition of biologic drugs does not appear to increase the morbidity rates of hepatic resection for colorectal liver metastases (29). In our series, no significant increase in postoperative complications was observed, with only 1 patient suffering from transient liver function impairment and no postoperative deaths. However, it has to be emphasized that, in this study, converted patients received a significantly lower number of cycles of chemotherapy than non-converted patients. Thus, conversion to surgery may be achieved after relatively few cycles of chemotherapy, thus preventing liver injury and severe post-operative complications.
The prognostic significance of synchronous versus metachronous disease remains controversial. Synchronous presentation has been reported to negatively affect long-term survival in some studies, whereas no differences in survival were found between colorectal cancer patients with early or delayed unresectable liver-only metastases in other investigations $(30,31)$. In our series, the relative risk of cancer mortality in metachronous metastases was $43 \%$ with respect to synchronous metastases. In addition, along with no chance of conversion, synchronous metastases were shown to be an independent prognostic variable of poor long-term outcome, as also confirmed by stepwise analysis.

In conclusion, our data support the role of anticancer treatments to shrink unresectable liver metastases from colorectal cancer. Surgery of liver metastases treated with conversion chemotherapy is the only way to significantly prolong outcome for this subset of patients. Prospective randomized trials are required to define the precise contribution of new therapeutic agents in these settings.

\section{References}

1. Poston GJ, Adam R, Alberts S, et al: OncoSurge: a strategy for improving resectability with curative intent in metastatic colorectal cancer. J Clin Oncol 23: 7125-7134, 2005.

2. Manfredi S, Lepage C, Hatem C, Coatmeur O, Faivre J and Bouvier AM: Epidemiology and management of liver metastases from colorectal cancer. Ann Surg 244: 254-259, 2006.

3. Sundermeyer ML, Meropol NJ, Rogatko A, Wang $\mathrm{H}$ and Cohen SJ: Changing patterns of bone and brain metastases in patients with colorectal cancer. Clin Colorectal Cancer 5: 108-113, 2005.

4. Nordlinger B, Van Cutsem E, Rougier P, et al: Does chemotherapy prior to liver resection increase the potential for cure in patients with metastatic colorectal cancer? A report from the European Colorectal Metastases Treatment Group. Eur J Cancer 43: 2037-2045, 2007.

5. Cook AD, Single R and McCahill LE: Surgical resection of primary tumors in patients who present with stage IV colorectal cancer: an analysis of surveillance, epidemiology, and end results data, 1988 to 2000. Ann Surg Oncol 12: 637-645, 2005.

6. Nordlinger B, Vauthey JN, Poston G, Benoist S, Rougier P and Van Cutsem E: The timing of chemotherapy and surgery for the treatment of colorectal liver metastases. Clin Colorectal Cancer 9: 212-218, 2010.

7. Tournigand C, André T, Achille E, et al: FOLFIRI followed by FOLFOX6 or the reverse sequence in advanced colorectal cancer: a randomized GERCOR study. J Clin Oncol 22: 229-237, 2004.

8. Geva R, Prenen H, Topal B, Aerts R, Vannoote J and Van Cutsem E: Biologic modulation of chemotherapy in patients with hepatic colorectal metastases: the role of anti-VEGF and anti-EGFR antibodies. J Surg Oncol 102: 937-945, 2010.

9. Galizia G, Lieto E, Orditura M, et al: First line chemotherapy vs bowel tumor resection plus chemotherapy for patients with unresectable synchronous colorectal hepatic metastases. Arch Surg 143: 352-358, 2008.

10. Galizia G, Castellano P, Pinto M, et al: Radiofrequency-assisted liver resection with a comb-shaped bipolar device versus clamp crushing: a clinical study. Surg Innov 19: 407-414, 2012.

11. Grothey A, Sargent D, Goldberg RM and Schmoll HJ: Survival of patients with advanced colorectal cancer improves with the availability of fluorouracil-leucovorin, irinotecan, and oxaliplatin in the course of treatment. J Clin Oncol 22: 1209-1214, 2004.

12. Van Cutsem E, Rivera F, Berry S, et al: Safety and efficacy of first-line bevacizumab with FOLFOX, XELOX, FOLFIRI and fluoropyrimidines in metastatic colorectal cancer: the BEAT study. Ann Oncol 20: 1842-1847, 2009.

13. Van Cutsem E, Köhne CH, Láng I, et al: Cetuximab plus irinotecan, fluorouracil, and leucovorin as first-line treatment for metastatic colorectal cancer: updated analysis of overall survival according to tumor $K R A S$ and $B R A F$ mutation status. J Clin Oncol 29: 2011-2019, 2011. 
14. Bokemeyer C, Bondarenko I, Hartmann JT, et al: Efficacy according to biomarker status of cetuximab plus FOLFOX-4 as first-line treatment for metastatic colorectal cancer: the OPUS study. Ann Oncol 22: 1535-1546, 2011.

15. Douillard JY, Siena S, Cassidy J, et al: Randomized, phase III trial of panitumumab with infusional fluorouracil, leucovorin, and oxaliplatin (FOLFOX4) versus FOLFOX4 alone as first-line treatment in patients with previously untreated metastatic colorectal cancer: the PRIME study. J Clin Oncol 28: 4697-4705, 2010.

16. Mitry E, Fields AL, Bleiberg H, et al: Adjuvant chemotherapy after potentially curative resection of metastases from colorectal cancer: a pooled analysis of two randomized trials. J Clin Oncol 26: 4906-4911, 2008.

17. Nordlinger B, Sorbye H, Glimelius B, et al: Perioperative chemotherapy with FOLFOX4 and surgery versus surgery alone for resectable liver metastases from colorectal cancer (EORTC Intergroup trial 40983): a randomised controlled trial. Lancet 371: 1007-1016, 2008.

18. Folprecht G, Grothey A, Alberts S, Raab HR and Köhne CH: Neoadjuvant treatment of unresectable colorectal liver metastases: correlation between tumour response and resection rates. Ann Oncol 16: 1311-1319, 2005

19. Masi G, Vasile E, Loupakis F, et al: Randomized trial of two induction chemotherapy regimens in metastatic colorecta cancer: an updated analysis. J Natl Cancer Inst 103: 21-30, 2011.

20. Folprecht G, Gruenberger T, Bechstein WO, et al: Tumour response and secondary resectability of colorectal liver metastases following neoadjuvant chemotherapy with cetuximab: the CELIM randomised phase 2 trial. Lancet Oncol 11: 38-47, 2010.

21. Klinger M, Tamandl D, Eipeldauer S, et al: Bevacizumab improves pathological response of colorectal cancer liver metastases treated with XELOX/FOLFOX. Ann Surg Oncol 17: 2059-2065, 2010.

22. Masi G, Loupakis F, Salvatore L, et al: Bevacizumab with FOLFOXIRI (irinotecan, oxaliplatin, fluorouracil, and folinate) as first-line treatment for metastatic colorectal cancer: a phase 2 trial. Lancet Oncol 11: 845-852, 2010.
23. Wong R, Cunningham D, Barbachano Y, et al: A multicentre study of capecitabine, oxaliplatin plus bevacizumab as perioperative treatment of patients with poor-risk colorectal liver-only metastases not selected for upfront resection. Ann Oncol 22: 2042-2048, 2011

24. Bertolini F, Malavasi N, Scarabelli L, et al: FOLFOX6 and bevacizumab in non-optimally resectable liver metastases from colorectal cancer. Br J Cancer 104: 1079-1084, 2011.

25. Adam R, Wicherts DA, de Haas RJ, et al: Complete pathologic response after preoperative chemotherapy for colorectal liver metastases: myth or reality? J Clin Oncol 26: 1635-1641, 2008.

26. Goèré D, Gaujoux S, Deschamp F, et al: Patients operated on for initially unresectable colorectal liver metastases with missing metastases experience a favorable long-term outcome. Ann Surg 254: 114-118, 2011.

27. Cleary JM, Tanabe KT, Lauwers GY and Zhu AX: Hepatic toxicities associated with the use of preoperative systemic therapy in patients with metastatic colorectal adenocarcinoma to the liver. Oncologist 14: 1095-1105, 2009.

28. Welsh FK, Tilney HS, Tekkis PP, John TG and Rees M: Safe liver resection following chemotherapy for colorectal metastases is a matter of timing. Br J Cancer 96: 1037-1042, 2007.

29. Pessaux P, Panaro F, Casnedi S, et al: Targeted molecular therapies (cetuximab and bevacizumab) do not induce additional hepatotoxicity: preliminary results of a case-control study. Eur J Surg Oncol 36: 575-582, 2010.

30. Robertson DJ, Stukel TA, Gottlieb DJ, Sutherland JM and Fisher ES: Survival after hepatic resection of colorectal cancer metastases: a national experience. Cancer 115: 752-759, 2009.

31. Rees M, Tekkis PP, Welsh FK, O'Rourke T and John TG: Evaluation of long-term survival after hepatic resection for metastatic colorectal cancer: a multifactorial model of 929 patients. Ann Surg 247: 125-135, 2008. 